\title{
IDENTIFICACIÓN DE IMPACTOSAMBIENTALES SIGNIFICATIVOS EN LA IMPLANTACIÓN DE PARQUES EÓLICOS. UN EJEMPLO EN EL MUNICIPIO DE JUMILLA (MURCIA)
}

\author{
José Molina Ruiz ${ }^{1}$ y M M Luz Tudela Serrano $^{2}$ \\ ${ }^{1}$ Suma Ambiental, S.L (Auditorías Ambientales). Murcia \\ ${ }^{2}$ Departamento de Geografía. Universidad de Murcia.
}

\section{RESUMEN}

Desde hace unos años se buscan en España soluciones a la dependencia respecto a los recursos energéticos no renovables. En nuestra Comunidad, la energía eólica se convierte en una energía alternativa real, conjuntamente con otras energías renovables, en especial, la solar fotovoltaica. El contar con una política de apoyo que favorece el desarrollo energético regional de una manera sostenible y la disponibilidad de zonas en nuestra región donde desarrollarla son la base de este trabajo, cuyo objetivo es la identificación de los emplazamientos idóneos para la instalación de parques eólicos en el municipio de Jumilla, considerando criterios ambientales.

Palabras clave: energía eólica, energías renovables, parque eólico.

\begin{abstract}
Since a few years ago in Spain is seeking solutions to the dependence on non-renewable energy resources. In our community, wind energy becomes a real alternative energy, along with other renewable energy sources, especially solar panel. Having a policy that aid the support regional energy development in a sustainable way and the availability of areas in our region develop where are the basis of this work, whose goal is the identification of sites suitable for the installation of wind parks in the municipality from Jumilla, considering environmental criteria.
\end{abstract}

Key words: wind energy, renewable energy, aeolian park.

\section{Antecedentes}

La energía eólica está experimentando en la actualidad una gran expansión sobre todo en Europa. Se trata de una tecnología muy antigua que ha vivido su mayor crecimiento 
a partir de la década de 1970 (debido a la crisis del petróleo de 1973) y, especialmente, a finales del siglo XX. Este crecimiento tan espectacular se debe fundamentalmente a la mejora del rendimiento energético y a una mayor potencia de generación, tanto en pequeños como en grandes sistemas eólicos, lo que redunda en un acercamiento entre la tecnología eólica y los sistemas de generación convencionales.

A pesar de que las plantas eólicas presentan un impacto ambiental relativamente pequeño en comparación con otras fuentes de energía convencionales, existe cierto rechazo principalmente por parte de los naturalistas. Las razones que se esgrimen son:

- El ruido producido por el giro del rotor, aunque su impacto no es más acusado que el generado por una instalación de tipo industrial de similar entidad.

- Impacto visual antiestético, ya que por sus características las centrales eólicas están localizadas generalmente en entornos naturales.

- Cientos de aves que mueren al colisionar con las palas de las turbinas.

- El elevado precio de la instalación. El aire al ser un fluido con peso específico muy bajo requiere de grandes máquinas y en consecuencia caras. Destacar el hecho de que el viento es una fuente de energía intermitente que no siempre está presente cuando se le necesita. Por tanto, el uso de la energía eólica para el autoabastecimiento de electricidad requiere de unos elementos acumuladores que encarecen la instalación.

La mayor parte de estos problemas se han solucionado o reducido mediante el desarrollo tecnológico o una adecuada ubicación de las plantas eólicas. En cualquier caso no podemos perder la perspectiva general y tener en cuenta que se trata de una energía «limpia», auspiciada desde las más altas instituciones europeas, nacionales y autonómicas, y que los impactos ambientales asociados son temporales y reversibles, siempre que en fase pre-operacional se diseñen las medidas correctoras adecuadas.

Con el presente trabajo se pretende obtener una valoración de los impactos potenciales que los Parques Eólicos pueden provocar sobre el paisaje y establecer las zonas viables, desde el punto de vista ambiental, para la instalación de los mismos en el Término Municipal de Jumilla (Murcia).

\section{Los impactos potenciales de la actividad}

Las operaciones que tienen lugar para llevar a cabo la instalación de Parques Eólicos pueden agruparse en cuatro fases, comprendiendo cada una de ellas una serie de acciones que ocasionan diferentes efectos sobre el medio:

\subsection{Fase de planificación del proyecto o pre-operacional}

En esta fase es preciso conocer con detenimiento el medio físico del entorno, investigación exhaustiva del recurso geológico, las figuras legales de protección ambiental, la viabilidad técnica y económica del proyecto y la incidencia social, como paso previo a su autorización (EIA). Resulta decisiva la determinación de los impactos potenciales (tabla 1) puesto que la magnitud del impacto dependerá en gran medida de la interacción existente entre el diseño del proyecto y de las medidas correctoras.

\subsection{Fase preparatoria/infraestructuras}

Incluye todos aquellos trabajos de dotación de infraestructuras. Las acciones causantes de impactos potenciales son: 
- Desbroce y retirada de la cubierta edáfica, movimiento de tierras para la construcción de accesos, viales e instalaciones.

- Construcción de las instalaciones auxiliares, red de evacuación eléctrica, caseta, etc.

Las alteraciones más significativas en esta fase del proyecto se traducirán en impactos sobre el suelo, la vegetación, la morfología y en impacto visual.

\section{C.- Fase operacional.}

Comprende el periodo de actividad de la estación eólica. Los impactos potenciales derivados del funcionamiento son impactos sobre la fauna e impacto visual.

\section{D.- Fase de abandono.}

Se trata de una actividad con una influencia reducida sobre su entorno, con una ubicación muy puntual, en la que no se generan vertidos ni emisiones contaminantes. Una vez que cesa la actividad los impactos generados son reversibles a corto plazo y concluirse que es respetuosa con el medio ambiente.

En esta fase se procederá a desmontar las instalaciones. Los impactos potenciales serán debidos al traslado de los equipos. Con el diseño de las medidas correctoras oportunas sobre el suelo, la vegetación y la morfología quedarán subsanados los impactos generados en las fases anteriores.

\section{Los impactos potenciales ambientales de la actividad}

\subsection{Impactos sobre el suelo}

El suelo es un recurso no renovable, sus características, su ubicación geográfica, su tiempo y hábitat determinados, son función de las influencias tanto naturales como humanas, comportándose como receptor de procesos múltiples que actúan modificando su naturaleza inicial (Tudela, 1993).

Las principales alteraciones del suelo que se pueden producir en las diferentes operaciones son las identificadas en la Tabla 1:

- Destrucción total o parcial del suelo.

- Compactación del suelo.

- Erosión del suelo.

- Contaminación del suelo.

\subsection{Impactos sobre la vegetación}

La cubierta vegetal tiene un papel primordial en la identificación de los impactos ambientales. La presencia de determinados tipos de plantas nos sirve de indicadores de las condiciones del medio natural, por ello se tienen muy en cuenta en la metodología para la identificación de impactos.

La vegetación se ve afectada por las distintas acciones del proyecto en las etapas preparatoria y operacional (tabla 1). El desarrollo del proyecto provoca la ocupación física del terreno con la construcción de edificios auxiliares, caminos de acceso, labores de desbroce y retirada de la cubierta edáfica. Los principales impactos potenciales sobre la vegetación derivados de la instalación son: 
- Eliminación de la cubierta vegetal.

- Degradación de las comunidades vegetales por presión antrópica.

- Pérdida de la vegetación clímax.

\subsection{Impactos sobre fauna}

Los principales impactos que tienen lugar sobre la fauna son los siguientes:

- Destrucción y cambios de tipo de hábitats: supone la desaparición de especies características de los ecosistemas por cambios en lugares de reposo, alimentación o refugio.

- Efecto barrera: ocasiona una disminución del tamaño poblacional de las especies, con el consiguiente riesgo de extinción, y una división del dominio vital de las especies.

- Eliminación o alteración de los biotopos terrestres.

- Modificación de pautas de comportamiento.

\subsection{Impacto visual}

El paisaje es una realidad compleja de definir puesto que existirán tantas realidades como puntos de vista desde donde se realicen. En todo caso, existe cierto consenso sobre el hecho de que en todo paisaje se pueden distinguir tres componentes: el espacio visual, la percepción de ese espacio y el hombre. El papel desempeñado por el hombre es fundamental, llegándose a decir que sin él no existiría el paisaje al no existir sujeto observador; por ello, el paisaje lo podemos entender como el conjunto de las formas naturales y antrópicas.

Un adecuado diseño del Proyecto minimizará en gran medida los impactos sobre el entorno, estableciendo un plan de trabajo adecuado, que contemple las medidas capaces de corregir el impacto visual en el futuro, impacto que se deriva del contraste de color, diseño de formas geométricas, etc. facilitando su reversibilidad a corto plazo.

A los factores físicos y bióticos perceptibles en que puede desagregarse el territorio se les denomina componentes del paisaje, que se corresponden con los impactos potenciales visuales causados por esta actividad:

- Aspecto de la superficie terrestre (relieve y morfología).

- El agua (redes de drenaje).

- La vegetación (tipo, distribución, densidad).

- Elementos artificiales (cultivos, carreteras, aerogeneradores, etc.).

Para lograr una aproximación objetiva al conocimiento del paisaje es preciso partir de una serie de conceptos como son: cuenca visual y fragilidad visual.

- Cuenca Visual se define como la superficie desde la que un punto es visible. Un concepto asociado es la intervisibilidad, que analiza el territorio en función del grado de visibilidad recíproca entre los diferentes puntos de la zona. Para definir la cuenca visual es preciso construir un Modelo Digital de Elevaciones a partir del cual poder obtener información sobre la morfología del territorio circundante al punto elegido de búsqueda. La definición de las cuencas visuales de diferentes puntos nos proporcionará información precisa para valorar los impactos visuales potenciales (Tudela y Molina, 2005)

— Fragilidad Visual se define como la susceptibilidad de un paisaje al cambio cuando se desarrolla una actividad sobre él; es decir, el potencial que un paisaje tiene para absorber una determinada acción humana, en este caso la implantación de Parques Eólicos (Tudela y Molina, 2002). 


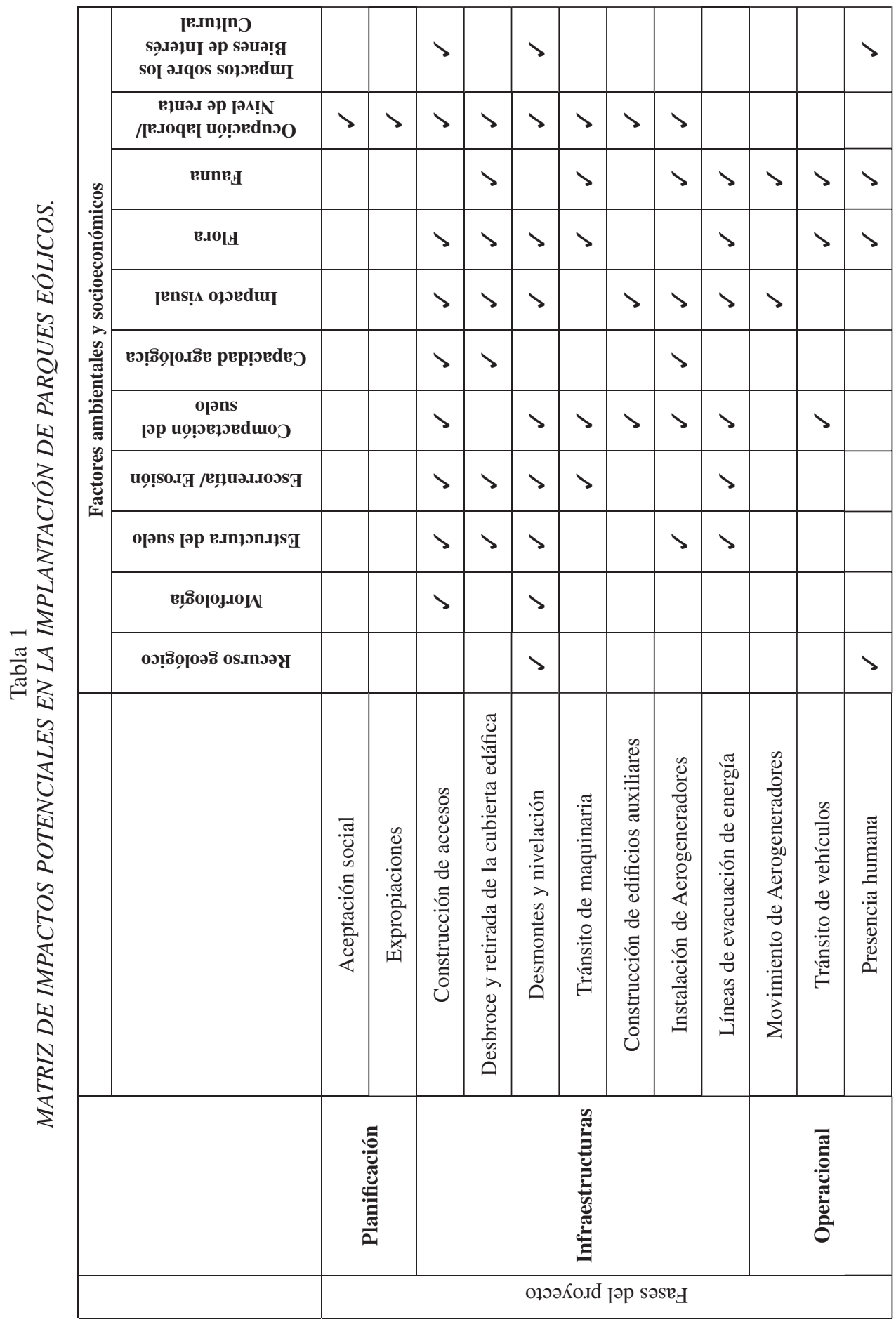


Se considera que la distancia ideal para el cálculo de cuencas visuales es de $10 \mathrm{~km}$ (Molina, et al., 2001), ya que a esta distancia el impacto potencial es alto, en cualquier condición de observación. Siguiendo esta argumentación se excluyen las cuencas visuales para mayores distancias porque en estos casos el impacto visual potencial es medio y bajo, y dependerá en gran medida de la variabilidad de las condiciones de observación, hecho que no puede ser modelizado.

\section{Resultados y conclusiones}

Siguiendo la metodología de identificación de impactos desarrollada se puede conocer la aptitud del medio para acoger los parques eólicos, partiendo del hecho de que las mejores condiciones son aquellas donde la fragilidad visual es menor. A modo de ejemplo, en este trabajo se establecen las zonas viables desde el punto de vista ambiental para la instalación de Parques Eólicos en el Término Municipal de Jumilla (Murcia).

El municipio de Jumilla se encuentra en el Noreste de la Región de Murcia y junto con Yecla constituyen la Comarca del Altiplano. Con una extensión de 970,6 km² , representa el $8,58 \%$ de la superficie regional (figura 1).

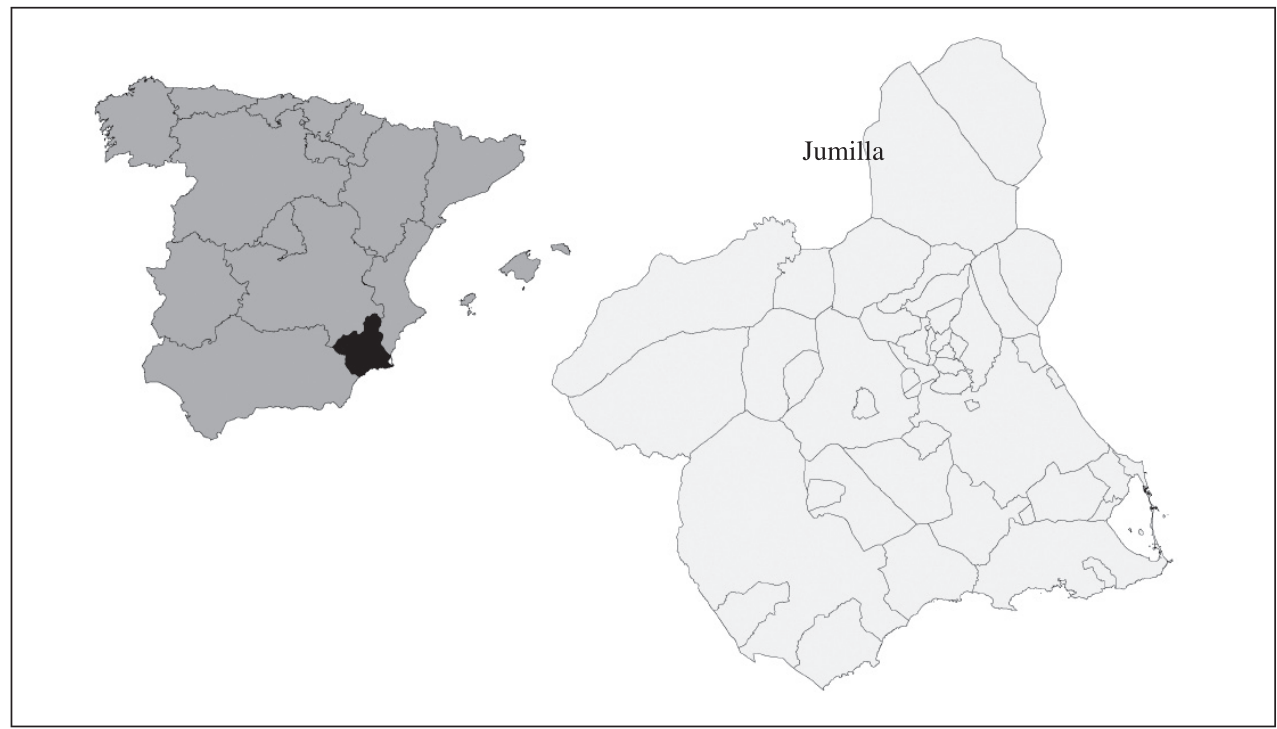

Figura 1. Zona de Estudio.

\subsection{Suelos}

Los suelos se han clasificado teniendo en cuenta los criterios de FAO-UNESCO (1988), resultando cinco unidades taxonómicas cartografiadas: Cambisoles, Fluvisoles, Litosoles, Regosoles y Xerosoles, a las que se les asigna diferentes grados de fragilidad en función de sus características edáficas (tabla 2). 
Tabla 2

RELACIÓN ENTRE SUELOS Y TERRITORIO OCUPADO

\begin{tabular}{|c|c|c|}
\hline Unidades de Suelos & Superficie Ocupada (\%) & Capacidad de Acogida \\
\hline Cambisoles & 4.16 & Baja \\
\hline Fluvisoles & 0.36 & Baja \\
\hline Litosoles & 30.25 & Alta \\
\hline Regosoles & 7.40 & Alta \\
\hline Xerosoles & 57.66 & Moderada \\
\hline
\end{tabular}

La instalación de Parques Eólicos supone una afección muy limitada sobre el suelo, ya que la superficie afectada se limita a la base de las torres de los aerogeneradores y a los caminos de acceso. Únicamente, Cambisoles y Xerosoles son los que presentan una mayor sensibilidad a la implantación de Parques Eólicos al ser los suelos de cultivo de la zona y contribuir en gran medida a la prosperidad económica. La pequeña extensión del área afectada supone que los impactos sobre el suelo ocasionados por esta actividad se consideran no significativos $\mathrm{y}$, en todo caso, reversibles con las adecuadas medidas de restauración.

\subsection{Vegetación}

En lo relativo a la vegetación se trata de un territorio alterado, la mayor parte del cual se ha visto sometido a una intensa antropización, con presencia de cultivos de secano y regadío. Existen algunas zonas que conservan su naturalidad en las sierras y otras áreas más reducidas en los valles, donde son frecuentes algunas especies protegidas de la Región de Murcia, como son los coscojares (Quercus coccifera) ricos en lentiscos (Pistacia lentis$c u s)$. Hay que tener en cuenta que vegetación y suelo presentan una dinámica convergente, aunque a distinta escala en el tiempo (tabla 3 ).

Tabla 3

RELACIÓN ENTRE COMUNIDADES VEGETALES Y TERRITORIO OCUPADO

\begin{tabular}{|c|c|c|}
\hline Comunidades vegetales & Superficie Ocupada (\%) & Capacidad de Acogida \\
\hline Pinares & 11.68 & Baja \\
\hline Matorrales & 6.62 & Moderada \\
\hline Espartal & 18.26 & Moderada \\
\hline Cultivos de Secano & 59.01 & Alta \\
\hline Cultivos de Regadío & 4.10 & Alta \\
\hline Áreas Periurbanas & 0.28 & Baja \\
\hline
\end{tabular}




\subsection{Fauna}

El inventario de la fauna requiere un trabajo de muestreo considerable. Los estudios son escasos y cuando los hay sólo aportan una referencia general. En el ámbito municipal se ha inventariado el Águila real, Buho real y Halcón peregrino que tienen su hábitat en distintas sierras, algunas de las cuales ya cuentan con figuras de protección ambiental.

En torno a los lugares de anidamiento se deben marcar unas áreas de exclusión cuya magnitud varía según las especies. Los criterios seguidos son los establecidos por la Dirección General del Medio Natural de la Consejería de Ordenación del Territorio y Medio Ambiente de la Región de Murcia:

Águila real: 750 metros.

Búho real: 500 metros.

Halcón peregrino: 500 metros.

Estas áreas en torno a los lugares de nidificación quedarán excluidas debido a su elevada sensibilidad y baja capacidad de acogida. En consecuencia, se considerarán no aptas para la instalación de Parques Eólicos.

\subsection{Paisaje}

Para determinar las cuencas visuales en el término municipal de Jumilla se consideró el criterio de seleccionar los puntos teniendo en cuenta su ubicación, que se corresponden con las zonas de mayor número de observadores potenciales, tomando siempre la mayor altitud. A mayor altitud mayor superficie visible desde el punto de referencia.

De acuerdo con los criterios descritos se han seleccionado dos puntos de la principal vía de comunicación que atraviesa el término municipal, la Nacional 334 Murcia-Valencia (puntos de referencia 1 y 2) (figura 2 A y B) y el núcleo urbano de Jumilla que aglutina la mayor concentración de población del territorio de estudio y, en consecuencia, reúne el mayor número de observadores potenciales. Siguiendo los criterios anteriores, se elige como punto de referencia, aquel con mayor altitud dentro de la ciudad (punto de referencia 3) (figura 2 C) (tabla 4).

A partir de los puntos de referencia citados se construyen los mapas «raster» de cuencas visuales con el programa de GIS Idrisi, definiendo un radio de búsqueda de $10 \mathrm{Km}$. La elaboración de estos mapas de cuencas visuales nos dará como resultado el Mapa de Fragilidad Visual del término municipal de Jumilla (figura 2 D).

Tabla 4

PUNTOS DE REFERENCIA PARA LA DETERMINACIÓN DE CUENCAS VISUALES

\begin{tabular}{|c|c|c|c|}
\hline \multirow{2}{*}{ Puntos de Referencia } & \multicolumn{3}{|c|}{ Coordenadas UTM } \\
\cline { 2 - 4 } & $\mathbf{X}$ & $\mathbf{Y}$ & $\mathbf{Z}$ \\
\hline 1.- CN-340 (PKM-83.5) & 653002.3 & 4270976 & $780 \mathrm{~m}$ \\
\hline 2.- CN-340 (PKM-62) & 652440.6 & 4255920 & $539 \mathrm{~m}$ \\
\hline 3.- Núcleo urbano de Jumilla & 646140.7 & 4260760 & $555 \mathrm{~m}$ \\
\hline
\end{tabular}

Aquellas zonas visibles desde los tres puntos de referencia, presentan una Fragilidad Visual Alta, lo que en la mayoría de los casos desaconseja la implantación de actividades. Aquellos lugares visibles desde dos de los tres puntos de vista de referencia tendrán una 


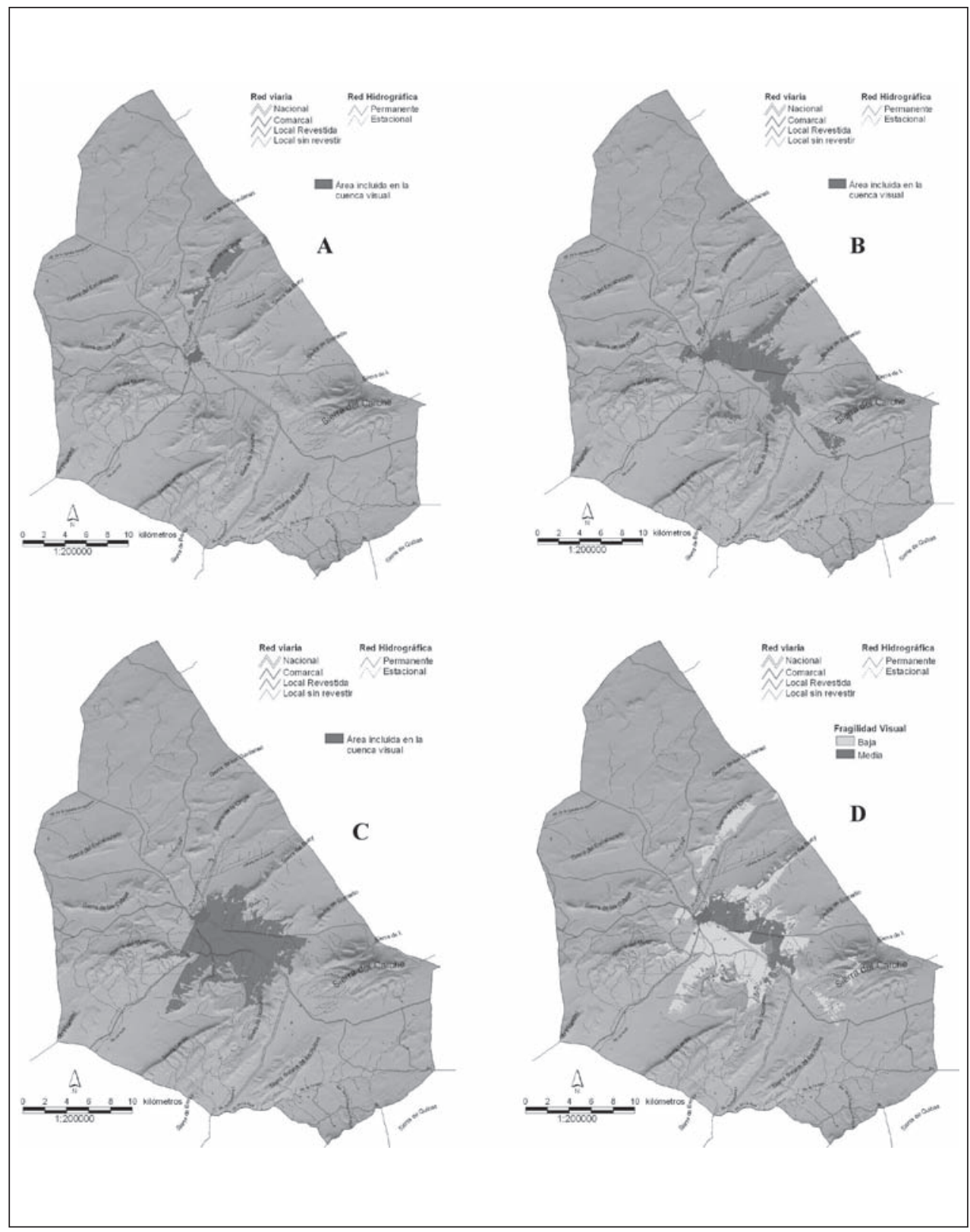

Figura 2. Cuencas visuales desde los tres puntos de referencia A B y C. Fragilidad Visual D 
Fragilidad Visual Media, la implantación de Parques Eólicos estará condicionada en función de las medidas correctoras adoptadas. Las zonas visibles sólo desde uno de los tres puntos de referencia considerados, se clasifican como de Fragilidad Visual Baja, por lo que la instalación de Parques Eólicos se considera compatible.

Del análisis del territorio no han resultado zonas con Fragilidad Visual Alta, lo que nos indica que no existen áreas de gran sensibilidad respecto al impacto visual en el término municipal. Las zonas de Fragilidad Visual Media ocupan el 2,15\% del término municipal $\left(20,95 \mathrm{~km}^{2}\right)$. En estas zonas la implantación de la actividad considerada es compatible con condiciones. Las zonas de Fragilidad Visual Baja suponen el 6,74 \% del territorio (65,48 $\mathrm{km}^{2}$ ), se corresponden con zonas muy concretas en torno a la ciudad de Jumilla y a las principales vías de comunicación. La implantación de Parques Eólicos se considera compatible pues el área afectada por el impacto visual es muy limitada en el territorio.

A través de la metodología de identificación de impactos desarrollada y validada satisfactoriamente en varios municipios de la Región de Murcia, se puede conocer la aptitud del medio para acoger los parques eólicos, partiendo del hecho que las mejores condiciones son aquéllas donde la fragilidad visual es menor.

\section{Bibliografía}

MOLINA, J. TUDELA, M.L.; CANO, Ma.P. Y BUENO, J.Mª (2001): «Minimización del impacto paisajístico en la actividad minera a cielo abierto. Demostración teórica y práctica de los costes de restauración». Papeles de Geografía, 33, 123-131. Universidad de Murcia.

DIRECTIVA79/409/CEE relativa a la conservación de las aves silvestres.

FAO-UNESCO (1988): World Soil Resources Report 60, FAO, París.

LEY 16/1985, de 25 de junio, de Patrimonio Histórico Español

LEY 4/89, de 27 de marzo, de conservación de los Espacios Naturales y de la Flora y la Fauna Silvestres.

REAL DECRETO 439/90 por el que se regula el Catálogo General de Especies Amenazadas.

LEY 4/92, de 30 de julio, de ordenación y protección del territorio de la Región de Murcia.

DIRECTIVA 92/43/CEE del Consejo, de 21 de mayo de 1992, relativa a la conservación de los hábitats naturales y de la fauna y flora silvestres

LEY 1/95, de 8 de marzo, de Protección del Medio Ambiente de la Región de Murcia.

LEY 7/95, de 21 de abril, de la Fauna Silvestre, Caza y Pesca Fluvial.

TUDELA, M.L. (1993): Estado actual de la degradación de suelos calizos en varias zonas de la Región de Murcia. Tesis Doctoral. Universidad de Murcia. 328 pp.

TUDELA, M.L. y MOLINA J. (2002): «Fragilidad visual de la actividad minera de roca ornamental en el municipio de Cehegín (Murcia)». Papeles de Geografía, 36, pp. 239-249. Universidad de Murcia.

TUDELA, M.L. y MOLINA J. (2005): «Estudio de viabilidad ambiental para la localización de parques eólicos en un municipio de la región de Murcia». Papeles de Geografía, 41-42, pp. 225236. Universidad de Murcia. 